\title{
The gut microbiota engages different signaling pathways to induce Duox2 expression in the ileum and colon epithelium
}

\author{
F Sommer ${ }^{1}$ and F Bäckhed ${ }^{1,2}$
}

The epithelium is a first line of defense against microorganisms in the gut. Reactive oxygen species (ROS) have an important role in controlling the normal gut microbiota and pathogenic bacteria. Dual oxidase 2 (DUOX2) is an important source of hydrogen peroxide in the small and large intestine, and the gut microbiota induces Duox2 expression. Here, we investigated the microbial regulation of Duox2 expression. We found that Duox2 was expressed by intestinal epithelial cells mainly in the tip of the epithelium. Duox2 expression was strongly induced by the presence of a normal microbiota in mice, but not when germ-free mice were colonized with various commensal bacteria. Duox2 expression was more rapidly induced by the gut microbiota in the colon than in the ileum. Furthermore, we showed that regulation of Duox2 expression in the ileum involved TIR-domain-containing adaptor protein including interferon- $\beta$ (TRIF) and canonical nuclear factor-кB p50/p65 signaling, whereas regulation of Duox2 expression in the colon involved MyD88 and the p38 pathway. Collectively, these data indicate that the gut microbiota uses two distinct signaling pathways to induce Duox2 expression in the ileum and colon epithelium.

\section{INTRODUCTION}

We live in close association with microorganisms (microbiota) that cover all surfaces of the body, with the majority residing within our intestinal tract. It is now recognized that the development of several increasingly abundant diseases such as obesity, atherosclerosis, and inflammatory bowel diseases is associated with dysbiosis of the microbiota. ${ }^{1}$ Thus, keeping a stable beneficial symbiosis seems to be a key requirement for health. ${ }^{2}$ The gut epithelium has an important role in controlling the normal gut microbiota through production of antimicrobial peptides and reactive oxygen species (ROS). ${ }^{3-7}$ The NADPH oxidase family members are the main ROSproducing enzymes ${ }^{3-7}$ and can be divided into two classes: NADPH oxidase and dual oxidase (DUOX) ${ }^{8,9}$ DUOX enzymes are characterized by their additional peroxidase homology domain, which exerts the catalytic function. ${ }^{10}$ Thus, DUOX enzymes produce hydrogen peroxide in a $\mathrm{Ca}^{2+}$-dependent manner, whereas most NADPH oxidase enzymes produce superoxide. ${ }^{11,12}$
Duox2 is expressed by surface epithelia in the lung or the intestine $e^{13,14}$ and its expression is increased during disease or dysbiosis, e.g., in patients with Crohn's disease or in response to Helicobacter pylori infections. ${ }^{15-17}$ In Drosophila, dDUOX (the homolog of mammalian DUOX2) produced ROS are required for protection from intestinal bacterial infection. ${ }^{18}$ However, DUOX2-mediated ROS production needs to be tightly regulated to avoid a nonspecific activation that would cause severe damage to the host tissue, e.g., during chronic inflammation. ${ }^{19}$

In Drosophila, dDUOX is regulated by the cooperative action of three signaling pathways: ${ }^{20}$ (i) a peptidoglycan pattern recognition receptor pathway, (ii) an unknown nonpeptidoglycan pattern recognition receptor signaling pathway and (iii) phospholipase $\mathrm{C}-\beta$ mediated inhibition of the p38 mitogen-activated protein kinase (MAPK) signaling pathway. In humans, DUOX2 activity is partially regulated via NOD2 signaling ${ }^{21}$ as well as by protein kinase C. ${ }^{22}$ In the mouse intestine, Duox 2 expression is induced by the microbiota, ${ }^{23}$ but it is not known

\footnotetext{
${ }^{1}$ The Wallenberg Laboratory and Sahlgrenska Center for Cardiovascular and Metabolic Research, Department of Molecular and Clinical Medicine, University of Gothenburg, Gothenburg, Sweden and ${ }^{2}$ Novo Nordisk Foundation Center for Basic Metabolic Research, Section for Metabolic Receptology and Enteroendocrinology, Faculty of Health Sciences, University of Copenhagen, Copenhagen, Denmark. Correspondence: F Bäckhed (Fredrik.Backhed@wlab.gu.se) 
how host-microbiota interactions regulate Duox 2 in mammals. Here, we investigated how the gut microbiota modulates Duox2 expression using germ-free (GF) and colonized mice, as well as genetically modified mice lacking components required for innate immune signaling. Finally, we used specific inhibitors of various signaling pathways in an in vitro cell culture system to identify molecular mechanisms of the host-microbiota crosstalk regulating Duox2 gene expression.

\section{RESULTS}

\section{Experimental colitis induced Duox2 expression}

DUOX2 levels are increased in patients with intestinal chronic inflammation or infections with $H$. pylori. ${ }^{15-17}$ To investigate whether Duox 2 expression is also modulated during chemically induced acute colitis, we treated mice with dextran sodium sulfate (DSS) and monitored Duox2 expression. Duox2 expression was immediately induced by the DSS treatment already one day after treatment and remained elevated during the course of colitis (Figure 1). Thus, Duox2 expression is rapidly modulated in response to injury and may thus contribute to disease.

\section{Duox2 is predominantly expressed by intestinal epithelial cells in the tip}

To address which cells in the epithelium express Duox2, we performed laser capture microdissection (LCM) followed by quantitative real-time PCR (qPCR) of different cellular fractions of the mouse intestinal tissue: tip and crypt epithelial

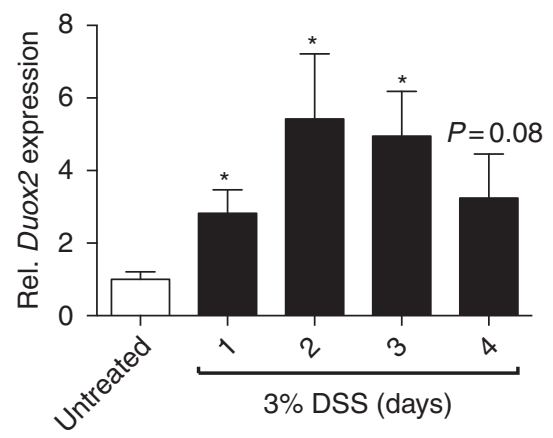

Figure 1 Duox2 expression is induced by experimental inflammation. Duox2 expression in the colon of C57BI6 wild-type mice during the time course of dextran sodium sulfate (DSS)-induced intestinal inflammation; $n=4$ per group. Data show mean \pm s.e.m.; ${ }^{*} P<0.05$ vs. untreated control. fractions from the ileum and colon of wild-type (WT) mice. First, we verified the purity of the fractions by assessing the expression of the tip-specific genes Alpi (alkaline phosphatase), Apoa4 (apolipoprotein A4), and Krt20 (keratin 20), and the crypt-specific genes Lyz1 (lysozyme 1), Defa5 (defensing alpha 5), Ki67 (antigen identified by monoclonal antibody Ki-67), and Ephb2 (ephrin B2), which were greatly enriched in the respective epithelial fractions (Supplementary Figure S1 online). Duox2 expression in the ileum was 190 times higher in the tip than in the crypt and hardly detectable in the mesenchymal fraction (Figure 2), which consists of mainly fibroblasts and immune cells. In the colon, Duox 2 expression was seven times higher in the tip than in the crypt (Figure 2). These data demonstrate that Duox2 expression is localized to the epithelium in the gut and predominantly to the differentiated epithelium in the ileum villus tip.

\section{The gut microbiota induces Duox2 expression in the intestine}

In a global microarray-based survey for microbially regulated genes, Duox 2 was identified as one of the genes that were most upregulated by the microbiota in the intestine. ${ }^{23}$ Interestingly, we found a significantly increased or trend toward higher peroxidase activity in ileal and colon protein lysates from conventionally raised (CONV-R) mice compared with GF mice, respectively (Figure 3a). Using qPCR, we verified that the gut microbiota induced Duox2 expression: Duox 2 levels were significantly higher in CONV-R compared with GF mice both in the ileum (ninefold) and colon (threefold) (Figure 3b). Similarly, Duox 2 expression was increased in CONV-R compared with GF mice in the duodenum and jejunum (Supplementary Figure S2), but their absolute expression levels were 10-100-fold lower as compared with those in the ileum and colon. Next, we determined Duox2 expression changes during colonization of GF mice with a normal microbiota. Duox2 expression kinetics differed in the ileum and colon (Figure 3c). In the ileum, a slight microbial induction of Duox2 expression was first detected 3 days after colonization) and expression increased to levels similar to CONV-R mice 7 and 14 days after colonization. In contrast, colonic Duox2 expression was readily induced by the gut microbiota already 1 day after colonization and increased to
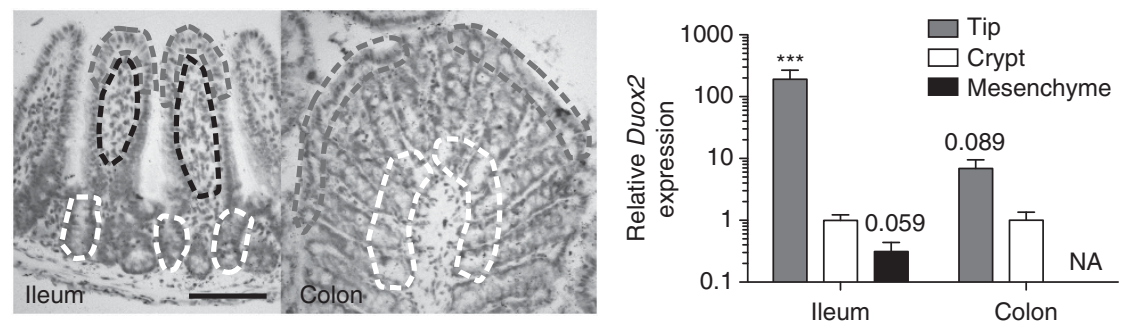

Figure 2 Duox2 is expressed by intestinal epithelial cells predominantly in the tip epithelium. Duox2 expression in laser capture microdissection (LCM)harvested tip (dashed red line) and crypt epithelial fractions (dashed white line) of the ileum and colon; $n=3-4$ per group. Data show mean $\pm \mathrm{s}$.e.m. and all values were normalized to expression in the ileum crypt; ${ }^{* \star *} P<0.001$ vs. crypt in the same tissue. A full color version of this figure is available at the Mucosal Immunology jounal online. 

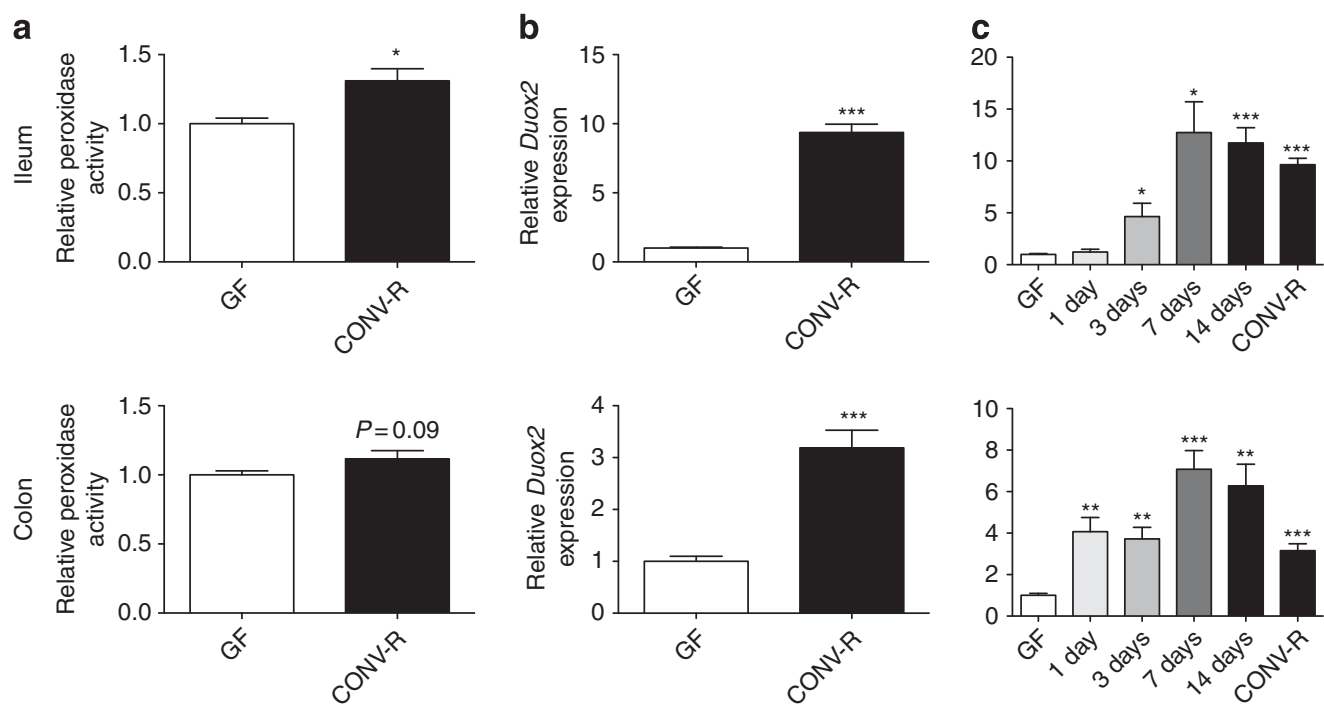

Figure 3 The gut microbiota induces Duox2 expression in the ileum and colon. (a) Relative peroxidase activity in the ileum and colon protein lysates of germ-free (GF) and conventionally raised (CONV-R) mice. ${ }^{*} P<0.05$. (b and $\left.\mathbf{c}\right)$ Duox2 expression (quantitative PCR) in the ileum and colon of (b) GF and CONV-R mice and (c) during colonization of GF mice with a normal microbiota; $n=6-7$ (a) and $n=4-5$ (b and c) per group. Data show mean \pm s.e.m.; ${ }^{\star} P<0.05,{ }^{* *} P<0.01$, and ${ }^{* *} P<0.001$ vs. GF.

levels higher than in CONV-R mice 7 and 14 days after colonization (Figure 3c). By measuring Tnfa expression, we tested whether Duox2 expression changes during colonization follow a transient inflammatory reaction, but no increases in Tnfa were detected (Supplementary Figure S3). We also investigated if Duox 2 expression could be induced by different commensals of the gut microbiota. None of the 13 tested bacterial strains ( 3 single bacteria or an assembly of 10 strains), including both Gram-positive and -negative bacteria, induced Duox2 expression (Supplementary Figure S4A and B) despite successful colonization (data not shown), suggesting that commensal-derived patterns are not sufficient to elicit Duox2 expression.

\section{Induction of Duox2 expression in the ileum involves NF-кB signaling}

Next, we aimed to determine the signaling pathways engaged to induce Duox2 expression in the intestinal epithelium. Therefore, we investigated Duox2 expression levels in mice deficient in different components of innate immune signaling, specifically the NF- $\kappa B$ pathway with the receptor adaptors TIR-domain-containing adaptor protein including interferon$\beta$ (TRIF) or MyD88 and the transcription factors $\mathrm{p} 50$ and $\mathrm{p} 65$. In the ileum, Duox2 expression was increased in $M y d 88^{-1-}$ mice and decreased in Trif ${ }^{-1-}$ and Myd88 $8^{-1-}$ Trif $^{-1-}$ compared with WT mice (Figure 4a, b). Furthermore, Duox2 expression involved canonical NF- $\mathrm{KB}$ signaling as expression was decreased in $p 50^{-1-}$ and IEC $p 65^{-1-}$ (VillinCre/p65 $5^{\mathrm{f} / \mathrm{f}}$ ) mice (Figure 4c). In contrast, Duox2 expression in the colon was decreased in $M y d 88^{-1-}$ mice compared with WT mice (Figure 4a), but increased in Trif $^{-1-}$ mice and unchanged in Myd88 $8^{-1-}$ Trif $^{-1-}, p 50^{-1-}$, and IEC $p 65^{-1-}$ mice (Figure $4 \mathbf{b}, \mathbf{c}$ ). These data indicate that Duox2 expression is partially induced via TRIF and p50/p65 in the ileum, and through the adaptor protein MyD88 in the colon.

\section{P38 pathway is required for IL-1 $\beta$-mediated Duox2 expression induction in the colon}

To investigate how Duox2 is regulated in the colonic epithelium, we treated ileal and colonic explants with the proinflammatory stimuli lipopolysaccharide (LPS) and interleukin (IL)-1 $\beta$, both of which are known to activate MyD88 signaling pathways. Only IL-1 $\beta$ induced Duox 2 expression in the ileum explants (Figure 5a), whereas both LPS and IL-1 $\beta$ induced Duox 2 expression in the colon explants (Figure $5 \mathbf{b}$ ). IL-1 $\beta$ and mouse intestinal content, but not LPS, also induced Duox2 expression in the human colon carcinoma cell line Caco-2 (Figure 5c, d). To confirm our qPCR data that NF- $\kappa \mathrm{B}$ is not involved in the regulation of Duox 2 expression in the colon, we generated luciferase-based reporter constructs with different stretches of the Duox2 promoter containing either WT or mutated NF- $\kappa B$ binding sites and transfected Caco- 2 cells with these vectors (Supplementary Figure S5A). Stimulation with IL-1 $\beta$ increased luminescence for the reporters containing the 1 or $3 \mathrm{~kb}$ constructs. However, mutation of the NF- $\kappa \mathrm{B}$ binding sites did not abolish the increase in luminescence by treatment with IL-1 $\beta$ (Supplementary Figure S5B), further demonstrating that Duox2 regulation in the colonic epithelium is independent of NF- $\mathrm{KB}$.

In an attempt to identify the signaling pathway leading to Duox2 expression in the colonic epithelium, we tested the effect of specific inhibitors of different signaling pathways downstream of MyD88 signaling on IL-1 $\beta$-induced increases in Duox2 expression in Caco- 2 cells. Pretreatment with the specific p38 MAPK inhibitor SB203580 (10 $\mu \mathrm{M}$ for $60 \mathrm{~min}$ ) abolished IL-1 $\beta$ mediated Duox2 expression (Figure 5e). No effect was observed 

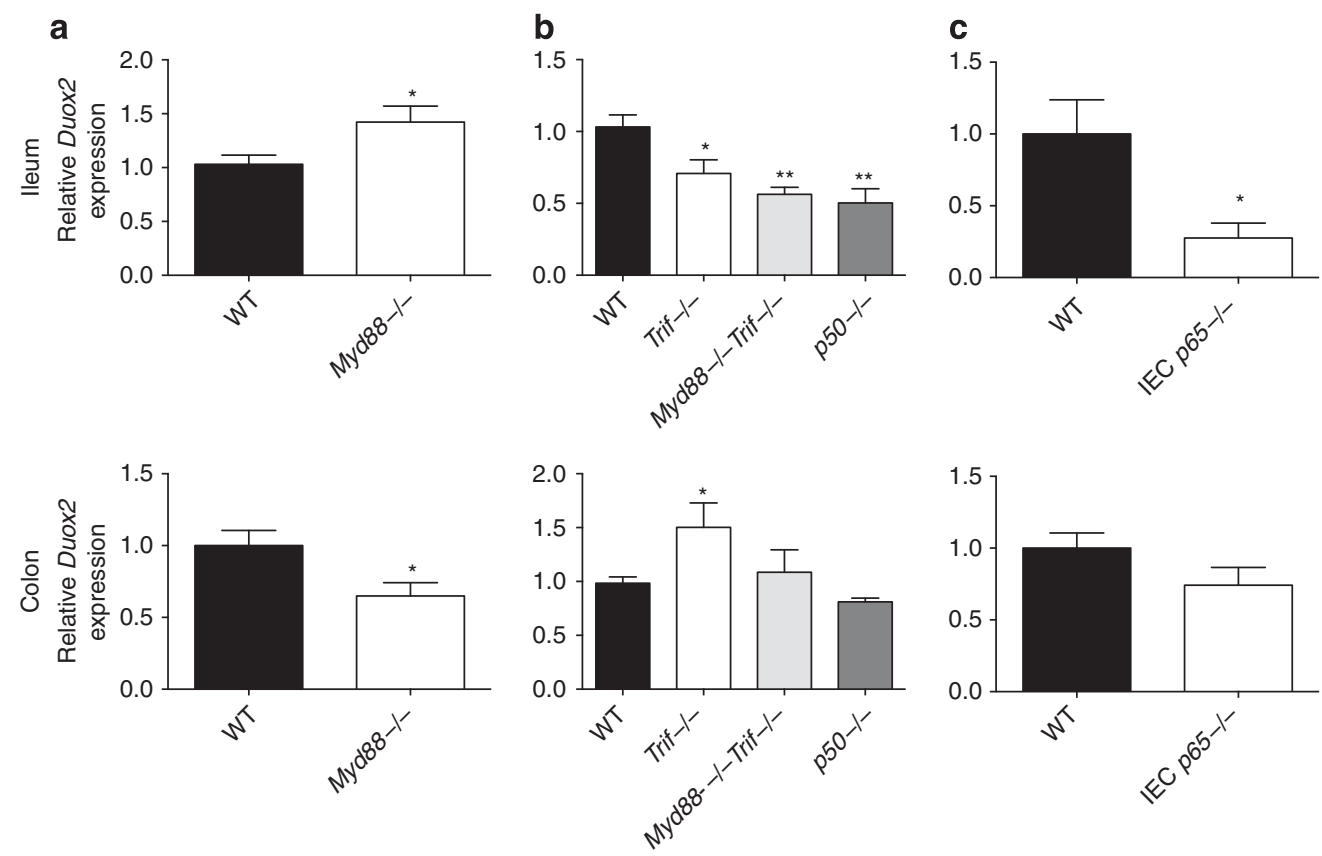

Figure 4 Microbial induction of Duox2 expression in the ileum involves nuclear factor- $\mathrm{kB}(\mathrm{NF}-\mathrm{\kappa B})$ signaling. Duox2 expression in the ileum and colon of (a) wild-type (WT) and Myd88 $8^{-1-}$ mice, (b) WT, Trif ${ }^{-1-}, M_{y d 88^{-1-}}$ Trif $^{-1-}$ compound homozygous knockouts, and p50 ${ }^{-1-}$ mice and (c) WT and IEC p65 (VillinCre/p65 ${ }^{\mathrm{f} / \mathrm{f}}$ ) mice; $n=4-15$ per group. Data show mean \pm s.e.m.; ${ }^{*} P<0.05$ and ${ }^{\star \star} P<0.01$ vs. WT.

with any of the other inhibitors (wedelolactone, IKK inhibitor; JSH-23, NF-אB activation inhibitor; SP600125, JNK-1/2/3 inhibitor; and U0126, MEK1/2 inhibitor). Importantly, pretreatment with SB203580 also abolished IL-1 $\beta$-mediated Duox2 expression in the colon explants (Figure 5f). Thus, our data suggest that Duox2 expression involves NF- $\kappa \mathrm{B}$ in the ileum and $\mathrm{p} 38$ in the colon.

\section{DISCUSSION}

We investigated the expression of Duox2 in the mouse intestinal tract and its regulation. Using LCM, we found that Duox2 was predominantly expressed in the tip epithelium of the ileum and colon and not in mesenchymal cells. In line with previous findings, ${ }^{23}$ Duox 2 expression was induced by a normal microbiota in both ileum and colon, but the expression kinetics differed in the two tissues: in the colon, Duox 2 expression was readily induced one day after colonization, whereas induction was delayed in the ileum, suggesting different regulatory pathways. In the ileum, microbial induction of Duox2 expression involved NF- $\mathrm{B}$ p50/p65 signaling through TRIF. In contrast, colonic Duox2 expression induction was mediated through MyD88 and p38 MAPK (Figure 6).

DUOX2 levels are increased in several inflammatory states including chronic intestinal inflammation or infections with H. pylori $;^{15-17}$ however, it is unknown whether DUOX2 is driving inflammation or activated in a secondary response. Our finding that DSS treatment immediately induced Duox2 expression argues for that DUOX2 may drive the inflammatory response as the induction occurred before any tissue damage. ${ }^{24}$ This hypothesis is further supported by our finding that changes in Duox2 expression during colonization of GF mice preceded those of Tnfa, a general marker for the inflammatory tone.

Duox2 expression kinetics during colonization differed greatly between the ileum and colon, which might reflect the different signaling pathways involved in these tissues. The delayed response in ileum could be due to indirect signaling toward a transient inflammatory reaction caused by colonization. However, no preceding increases in inflammatory markers were detected. Instead, we speculate that epithelial cells of the small intestine are resistant to stimuli after leaving the crypt to avoid excessive immune reactions toward the microbiota, as they are not covered by a thick mucus layer that would inhibit contact to the resident bacteria. This hypothesis is supported by the finding that only basolateral stimuli are capable of activating Toll-like receptor 5 (TLR5) or TLR9 proinflammatory signaling in polarized intestinal epithelial cells, thus limiting responsiveness only to microbes that penetrate the epithelial barrier. ${ }^{25,26}$ Another possible explanation could be that we used a cecal inoculum for colonization, which might be more adapted to the colon habitat and thus it could take longer for the microbiota to adapt to the ileum and induce proper signaling.

Duox2 expression levels were unaffected by colonization with 13 tested commensals including Escherichia coli, Bifidobacterium longum, or Bacteroides thetaiotaomicron. Thus, it seems unlikely that a single microbe-associated molecular pattern could be the stimulus. It is known that Duox2 expression is stimulated by several pathogens including Listeria monocytogenes or Helicobacter felis or in patients with inflammatory bowel disease, which is associated with an increase of pathogens. ${ }^{21,27}$ However, these bacteria are not present in our 
a

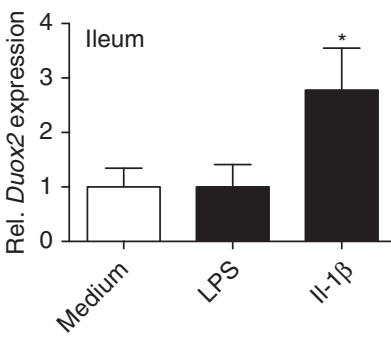

C

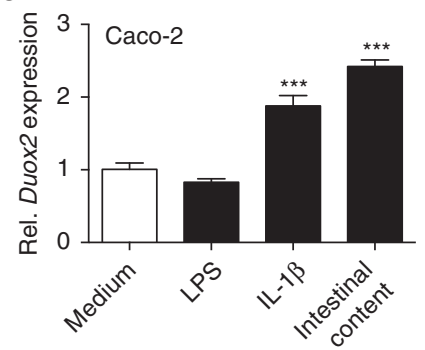

e
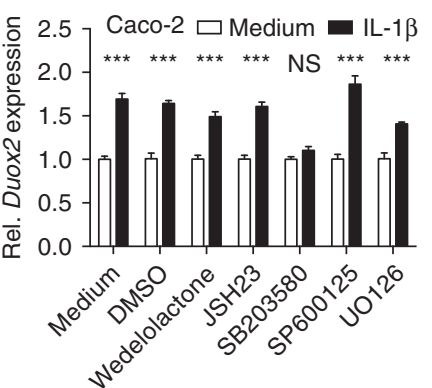

b

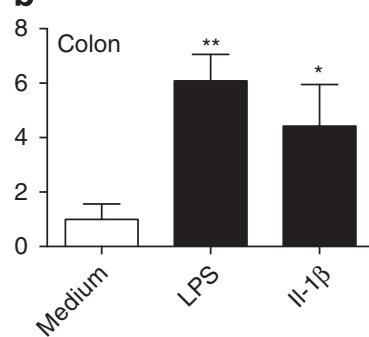

d

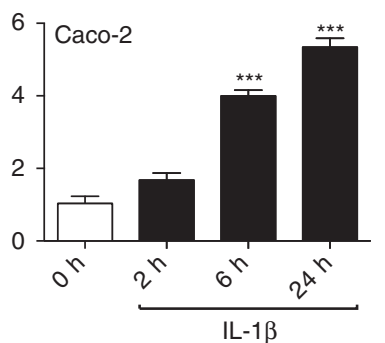

f

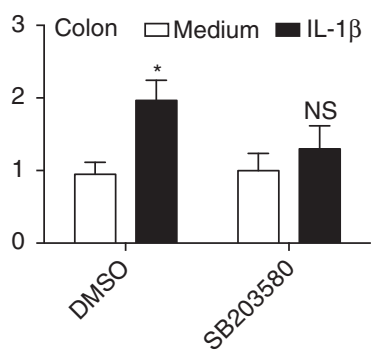

Figure 5 p38 pathway is required for IL-1 $\beta$-induced Duox2 expression induction in the colon. Duox2 expression after incubation with $100 \mathrm{ng} \mathrm{ml}^{-1}$ lipopolysaccharide (LPS) or $20 \mathrm{ng} \mathrm{ml}^{-1}$ interleukin-1 $\beta$ (IL-1 $\beta$ ) (a) for $24 \mathrm{~h}$ in the ileum explant, (b) for $24 \mathrm{~h}$ in the colon explant. (c) Duox2 expression after incubation with $100 \mathrm{ng} \mathrm{ml}^{-1} \mathrm{LPS}, 20 \mathrm{ng} \mathrm{ml}^{-1} \mathrm{IL}-1 \beta$, or $10 \%$ sterile filtered intestinal content for $6 \mathrm{~h}$ in Caco-2 cells. (d) Duox2 expression in Caco-2 cells after incubation with $20 \mathrm{ng} \mathrm{ml}^{-1} \mathrm{IL}-1 \beta$ for 2,6 , or $24 \mathrm{~h}$. (e) Duox2 expression in Caco-2 cells treated with specific inhibitors of IL-1 $\beta$ downstream signaling cascades before stimulation with IL-1 $\beta$. Inhibitors were wedelolactone (IKB kinase (IKK) inhibitor, $50 \mu \mathrm{m}$ for $60 \mathrm{~min}$ ), $\mathrm{JSH}-23$ (NF- $\kappa B$ activation inhibitor, $10 \mu \mathrm{M}$ for $60 \mathrm{~min}$ ), SB203580 (p38 mitogenactivated protein kinase (MAPK) inhibitor, $10 \mu \mathrm{m}$ for $60 \mathrm{~min}$ ), SP600125 (c-Jun N-terminal kinase (JNK)-1/2/3 inhibitor, $50 \mu \mathrm{m}$ for $45 \mathrm{~min}$ ) and U0126 (MAP kinase kinase (MEK1/2) inhibitor, $10 \mu \mathrm{m}$ for $2 \mathrm{~h}$ ). All inhibitors were dissolved in dimethyl sulfoxide (DMSO). (f) Duox2 expression in the colon explants treated with DMSO or SB203580 before stimulation with IL$1 \beta$ for $24 \mathrm{~h}$; $n=3-5$ per group. Data show mean \pm s.e.m.; ${ }^{\star} P<0.05$, ${ }^{\star \star} P<0.01$, and ${ }^{* \star *} P<0.001$ vs. medium.

animal facility and thus cannot explain why our normal specific pathogen-free microbiota induces Duox 2 expression in the absence of inflammation. It is possible that only a limited number of bacteria (different from the ones we tested) might be able to induce Duox2 expression. Alternatively, the bacterial density may be needed to be higher or specific metabolites may be required to induce Duox2 expression.

Duox2 expression was differentially regulated in the ileum and colon. We noted that signaling in the ileum was partially mediated via NF- $\kappa$ B and TRIF, whereas Myd88 and p38 were

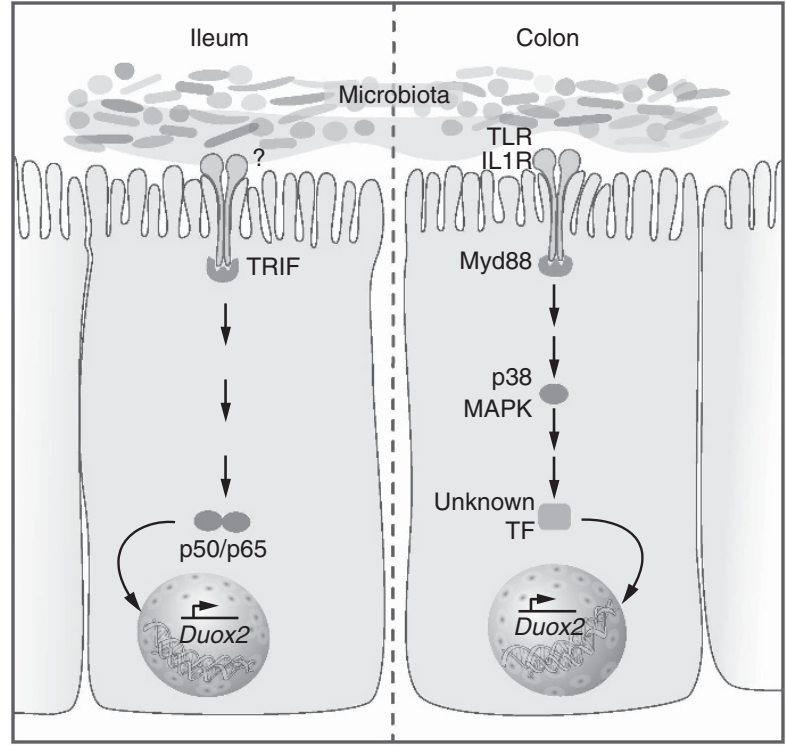

Figure 6 Model for the differential microbial regulation of Duox2 expression in the ileum and colon. In the ileum the microbial signal might be sensed by an unknown receptor and transmitted through TIR-domaincontaining adaptor protein including interferon- $\beta$ (TRIF) and p50/p65 initiating Duox2 expression. In the colon the microbial signal might be recognized by a TLR or IL-1 receptor and transmitted through MyD88, p38 MAPK and a yet unknown non-p50/p65 transcription factor (TF) leading to Duox2 expression.

involved in the colon. There are several differences in these tissues that could account for the differential regulation. For example, the microbiota composition differs between the ileum and colon, ${ }^{1}$ and therefore the microbiota signals inducing Duox2 expression could differ in these tissues. Moreover, the cellular composition, ${ }^{28}$ tissue architecture, ${ }^{29}$ and functional metabolic properties ${ }^{30}$ differ between the ileum and colon.

Both LPS and IL-1 $\beta$ activate MyD88 signaling pathways and induced Duox 2 expression in the colon explants. However, in Caco- 2 cells IL-1 $\beta$, but not LPS, was capable of inducing Duox2. This differential response in the Caco- 2 cells might be because they are a cancer cell line and thus do not exactly recapitulate primary intestinal epithelial cells. The prototypical receptors for LPS and IL-1 $\beta$ are TLR4 and the IL-1 receptor, respectively. ${ }^{31,32}$ Only TLR3 or TLR4 signal through TRIF, whereas all TLRs (except TLR3) and the IL-1 and IL-18 receptors signal through MyD88. Thus, our data suggest that in the ileum TLR3 or TLR4 and TRIF and in the colon TLRs and MyD88 may be involved in the regulation of Duox2. However, in Myd88 $8^{-1-}$ Trif $^{-1-}$ mice, which completely lack pattern recognition receptor signaling, Duox2 expression was not completely abolished, indicating the presence of another signaling mechanism. Our findings are supported by recent findings showing that in human pancreas cell lines TLR4 is required to induce Duox $2^{33}$ and that some NADPH oxidase family members interact physically with components of the TLR signaling cascade. ${ }^{34-36}$ However, we cannot exclude that the gut microbiota induces Duox2 expression indirectly 
through a pathway that involves host production of IL-1 or IL-18, which also mediate signaling from their receptors through MyD88. Furthermore, we cannot rule out effects of non-epithelial cells in the signaling leading to increased Duox2 expression but analyzing conditional intestinal-specific knockout mice could clarify this.

Microbiota-induced Duox2 expression may at first glance seem useful for the host but undesirable for the bacteria as ROS are antibacterial. However, not all bacteria show the same susceptibility to ROS. ${ }^{37,38}$ Thus, one hypothesis could be that those bacteria that induce Duox2 expression might also be more resistant to ROS, thereby exploiting a host defense system to reduce competition by other more susceptible bacteria. Furthermore, it has been shown that commensal bacteria tone down proinflammatory NF- $\mathrm{KB}$ signaling through ROS. ${ }^{39,40}$ However, the origin of these ROS is not clear. If these ROS originate from host DUOX2 induced by the microbiota, they could counteract their direct antibacterial effects and instead be beneficial for the microbes by toning down other inflammatory responses.

Taken together, here we found that in the mouse expression of Duox2 is differentially induced by the microbiota and regulated via two different signaling pathways in the ileum and colon. These findings highlight the need for further studies delineating host microbial interactions in the gut.

\section{METHODS}

Mice. C57Bl6/J and Swiss-Webster mice, as well as mice lacking

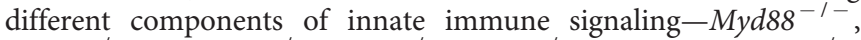
Trif $^{--1-}$, MyD $88^{-1-}$ Trif $^{--1-}, p 50^{-1-}$, and IEC $p 65^{-1-}$ (VillinCre/p65 $5^{\mathrm{f} / \mathrm{f}}$ ) -were maintained under standard specific pathogen-free or GF conditions as described previously. ${ }^{24}$ All mice were 10 15 weeks of age and sex and age matched for individual experiments. GF mice were colonized with a normal cecal microbiota by oral gavage and maintained for up to 14 days as described. ${ }^{41}$ A similar protocol was used to colonize GF mice with single bacterial species Escherichia coli, Bifidobacterium longum, or Bacteroides thetaiotaomicron or with an assembly of 10 commensal bacteria (Petia Kovetcheva, personal communication). The mice colonized with the 10 commensal bacteria were bred in isolators for at least eight generations before use in an experiment. Mice were killed by cervical dislocation and the distal part of the small intestine (ileum) and the proximal part of the colon were removed and frozen in liquid nitrogen. Animal protocols were approved by the Gothenburg Animal Ethics Committee.

DSS treatment. Colonic inflammation was induced in 9-14-week-old mice by administration of 3\% DSS (TdB TdB Consultancy, Uppsala, Sweden) for four days in drinking water as described previously. ${ }^{42}$ Colitis severity was assessed as described and presented elsewhere. ${ }^{24}$

Laser capture microdissection. C57Bl6/J female mice were used at 12 weeks of age. LCM was performed as described previously. ${ }^{43}$ Briefly, mice were killed by cervical dislocation and the intestine removed. The ileum and the proximal part of the colon were excised, flushed with phosphate-buffered saline, and finally flushed and embedded with OCT freezing medium. Eight-micrometer-thick cryosections were cut using a microtome (Leica, Nussloch, Germany), placed onto PEN membrane slides (Zeiss, Jena, Germany), and stained immediately. After dehydration in absolute and $70 \%$ ethanol, OCT was removed by dipping in RNase-free water before staining with $1 \%$ cresyl violet in $50 \%$ ethanol. Slides were partially destained by dipping in $70 \%$ and absolute ethanol before air-drying. Slides were stored in airtight containers at $-80^{\circ} \mathrm{C}$ until LCM. LCM was performed using PALM Microbeam Microdissection microscope (Zeiss) and fractions were collected dry. The harvested fractions were immediately lysed in RLT (a guanidine-thiocyanate-containing lysis buffer) containing 1\% $\beta$ mercaptoethanol and stored at $-80^{\circ} \mathrm{C}$. RNA was isolated from the lysates using RNeasy Micro Kit (Qiagen, Hilden, Germany). The complete RNA volume was used for synthesis of cDNA before qPCR.

Preparation of sterile mouse intestinal content. Intestinal content from almost the complete intestine of one CONV-R donor mouse (from jejunum until distal colon) was mixed with $15 \mathrm{ml}$ phosphatebuffered saline. After centrifugation, the supernatant was passed through a $0.2 \mu \mathrm{m}$ filter.

Peroxidase activity. Peroxidase activity was measured as described previously. ${ }^{44}$ Briefly, ileum or colon tissue was homogenized in RIPA buffer (50 mм Tris-HCl, pH 7.4, $150 \mathrm{~mm} \mathrm{NaCl}, 1 \%$ Nonidet P-40, 0.5\% sodium deoxycholate, $0.1 \%$ sodium dodecyl sulfate). Fifty micrograms of protein was added to $100 \mu \mathrm{l}$ TMB solution (Sigma, St Louis, MO). After incubation at room temperature for $30 \mathrm{~min}$, absorbance at $650 \mathrm{~nm}$ was recorded.

Expression analysis using q PCR. Duodeum, jejunum, ileum, or colon tissue $(30 \mathrm{mg})$ was homogenized with TissueLyser (Qiagen, Hilden, Germany), and total RNA was isolated with the RNeasy Mini Kit (Qiagen). The High Capacity cDNA Reverse Transcription Kit (Applied Biosystems, Foster City, CA) was used to synthesize $20 \mu \mathrm{l}$ cDNA templates from $500 \mathrm{ng}$ purified RNA with random hexamer primers, and the products were diluted seven times before use in subsequent reactions. SYBR Green Master Mix buffer $(1 \times$; Bio-Rad Laboratories) was used for q PCR at final reaction volumes of $25 \mu \mathrm{l}$. Gene-specific results were normalized to the ribosomal protein L32 mRNA (primer sequences can be found in Supplementary Table S1). Assays were performed in a CFX96 Real-Time System (Bio-Rad Laboratories). The reactions were analyzed with the $\Delta \Delta \mathrm{CT}$ analysis method.

Ex vivo stimulation. Mice were killed by cervical dislocation and a $2 \mathrm{~cm}$ colon segment was collected. Intestinal contents were removed by flushing with phosphate-buffered saline and the segment was divided into four equal parts. Each part was placed in 24 -well cell culture plates containing the growth medium (Dulbecco's modified Eagle's medium with glutamine and pyruvate, $4.5 \mathrm{gl}^{-1}$ glucose, $10 \%$ fetal calf serum, $100 \mathrm{U} \mathrm{ml}^{-1}$ penicillin, and $100 \mathrm{mg} \mathrm{ml}^{-1}$ streptomycin) and either LPS $\left(100 \mathrm{ng} \mathrm{ml}^{-1}\right)$ or IL-1 $\beta\left(20 \mathrm{ng} \mathrm{ml}^{-1}\right)$. The plates were then incubated overnight at $37^{\circ} \mathrm{C}$ with $5 \% \mathrm{CO}_{2}$. The content of each well was transferred to Eppendorf tubes and centrifuged briefly. The tissue pellet was used for the analysis of mRNA expression.

Caco-2 cell culture. Human colon carcinoma cells (Caco-2; clone HTB-37) were purchased from ATCC (Manassas, VA) and maintained according to the supplier's instructions. Briefly, cells were grown in Eagle's minimum essential medium supplemented with $20 \%$ fetal bovine serum, $2 \mathrm{~mm}$ L-glutamine, $0.1 \mathrm{~mm}$ nonessential amino acids, $1.5 \mathrm{gl}^{-1}$ sodium bicarbonate, $1.0 \mathrm{~mm}$ sodium pyruvate, and $1 \%$ penicillin-streptomycin antibiotic solution. Cells were passaged at $80 \%$ confluency and seeded into 24 -well plates for experiments. Cells were either incubated with LPS ( $100 \mathrm{ng} \mathrm{ml}^{-1}$ ) for $6 \mathrm{~h}$ or with IL- $1 \beta$ $\left(20 \mathrm{ng} \mathrm{ml}^{-1}\right.$ ) for 2,6 , or $24 \mathrm{~h}$ or mouse intestinal content (10\%) for $6 \mathrm{~h}$. For inhibitor experiments, cells were pretreated with indicated specific inhibitors of different IL- $1 \beta$ downstream signaling cascades before stimulation with $20 \mathrm{ng} \mathrm{ml}^{-1}$ IL-1 $\beta$ for $6 \mathrm{~h}$. The inhibitors were wedelolactone (IKK inhibitor, $50 \mu \mathrm{M}$ for $60 \mathrm{~min}$ ), JSH-23 (NF- $\mathrm{KB}$ activation inhibitor, $10 \mu \mathrm{M}$ for $60 \mathrm{~min}$ ), SB203580 (p38 MAPK inhibitor, $10 \mu \mathrm{M}$ for $60 \mathrm{~min}$ ), SP600125 (JNK-1/2/3 inhibitor, $50 \mu \mathrm{M}$ for $45 \mathrm{~min}$ ), and U0126 (MEK1/2 inhibitor, $10 \mu \mathrm{M}$ for $2 \mathrm{~h}$ ). All inhibitors were dissolved in dimethyl sulfoxide. Medium was removed and the cells were lysed using RLT containing $1 \% \beta$-mercaptoethanol directly in the wells. Lysates were then used for analysis of mRNA expression. 
Luciferase reporter system. Luciferase reporter constructs were generated using a modified pGL4.17 reporter vector (Promega, Madison, WI) and 1 or $3 \mathrm{~kb}$ of the Duox 2 promoter. Putative NF-kB binding sites were predicted using ConSite ${ }^{45}$ and mutated using GENEART Site-Directed Mutagenesis System (Invitrogen, Carlsbad, CA) according to the manufacturer's instructions. Primer sequences can be found in Supplementary Table S1. Sequences of the reporter constructs have been submitted to GenBank (accession numbers KJ408274-KJ408277). Caco-2 cells were stably transfected with the different Duox2::luciferase reporter constructs using Lipofectamine (Invitrogen) and successive $\mathrm{G} 418$ (Invitrogen; $0.4 \mathrm{mg} \mathrm{ml}^{-1}$ ) selection. In all, 500,000 cells per well were seeded into a 24 -well plate and grown overnight to confluency. Cells were incubated with $20 \mathrm{ng} \mathrm{ml}^{-1}$ IL-1 $\beta$ for $6 \mathrm{~h}$ and lysed in Glo Lysis buffer (Promega). After mixing the lysate with Steady-Glo luciferase reagent (Promega), luminescence was measured using GloMax 20/20 luminometer (Turner BioSystems, Sunnyvale, CA).

Statistical analysis. Data are presented as mean \pm s.e.m. Statistical differences between groups of two were analyzed with a Student's $t$-test, comparisons of three or more groups with one independent variable (e.g., genotype) were analyzed by one-way analysis of variance with ad hoc Bonferroni post hoc tests using Prism 5 (GraphPad Software, La Jolla, CA).

Supplementary Material is linked to the online version of the paper at http://www.nature.com/mi

\section{ACKNOWLEDGMENTS}

We thank Rosie Perkins for editing the manuscript. We are grateful to Nina Adam and Bilal Bayazit for experimental assistance, Manolis Pasparakis and Andy Wullaert for providing tissues from IEC $p 65^{-1-}$ mice, Carina Arvidsson, Sara Nordin-Larsson, Ulrica Enqvist, and Lovisa Källman for animal husbandry, Petia Kovatcheva and Valentina Tremaroli for providing samples from mice colonized with commensal bacteria species, and Anna Hallén for help with the artwork. We are indebted to the staff at Centre for Cellular Imaging of the University of Gothenburg for superb technical assistance with the LCM procedure. This study was supported by the Swedish Research Council, Torsten and Ragnar Söderbergs' foundations, IngaBritt and Arne Lundberg's foundation, Swedish Foundation for Strategic Research, Knut and Alice Wallenberg foundation, and the regional agreement on medical training and clinical research (ALF) between Region Västra Götaland and Sahlgrenska University Hospital.

\section{DISCLOSURE}

FB is founder and owns equity in Metabogen AB. The remaining author declared no conflict of interest.

c 2015 Society for Mucosal Immunology

\section{REFERENCES}

1. Sommer, F. \& Backhed, F. The gut microbiota-masters of host development and physiology. Nat. Rev. Microbiol. 11, 227-238 (2013).

2. Garrett, W.S., Gordon, J.I. \& Glimcher, L.H. Homeostasis and inflammation in the intestine. Cell 140, 859-870 (2010).

3. Babior, B.M. The respiratory burst oxidase. Curr. Opin. Hematol. 2, 55-60 (1995).

4. Klebanoff, S.J. Role of the superoxide anion in the myeloperoxidasemediated antimicrobial system. J. Biol. Chem. 249, 3724-3728 (1974).

5. Pullar, J.M., Vissers, M.C. \& Winterbourn, C.C. Living with a killer: the effects of hypochlorous acid on mammalian cells. IUBMB Life 50, 259-266 (2000).

6. Quinn, M.T. \& Gauss, K.A. Structure and regulation of the neutrophil respiratory burst oxidase: comparison with nonphagocyte oxidases. J. Leukoc Biol. 76, 760-781 (2004)

7. Rosen, H., Crowley, J.R. \& Heinecke, J.W. Human neutrophils use the myeloperoxidase-hydrogen peroxide-chloride system to chlorinate but not nitrate bacterial proteins during phagocytosis. J. Biol. Chem. 277, 30463-30468 (2002).
8. Bokoch, G.M. \& Knaus, U.G. NADPH oxidases: not just for leukocytes anymore!. Trends Biochem. Sci. 28, 502-508 (2003).

9. Lambeth, J.D. NOX enzymes and the biology of reactive oxygen. Nat. Rev. Immunol. 4, 181-189 (2004).

10. Lambeth, J.D., Kawahara, T. \& Diebold, B. Regulation of Nox and Duox enzymatic activity and expression. Free Radic BiolMed. 43, 319-331 (2007).

11. Bjorkman, $\mathrm{U}$. \& Ekholm, R. Generation of $\mathrm{H}_{2} \mathrm{O}_{2}$ in isolated porcine thyroid follicles. Endocrinology 115, 392-398 (1984).

12. Dupuy, C., Kaniewski, J., Deme, D., Pommier, J. \& Virion, A. NADPHdependent $\mathrm{H} 2 \mathrm{O} 2$ generation catalyzed by thyroid plasma membranes. Studies with electron scavengers. Eur. J. Biochem. 185, 597-603 (1989).

13. Krause, K.H. Tissue distribution and putative physiological function of NOX family NADPH oxidases. Jpn J. Infect. Dis. 57, S28-S29 (2004).

14. El Hassani, R.A. et al. Dual oxidase2 is expressed all along the digestive tract. Am. J. Physiol. Gastrointest Liver Physiol. 288, G933-G942 (2005).

15. Csillag, C. et al. Expression of the genes dual oxidase 2, lipocalin 2 and regenerating islet-derived 1 alpha in Crohn's disease. Scand. J. Gastroenterol. 42, 454-463 (2007).

16. Rokutan, K., Kawahara, T., Kuwano, Y., Tominaga, K., Nishida, K. \& Teshima-Kondo, S. Nox enzymes and oxidative stress in the immunopathology of the gastrointestinal tract. Semin. Immunopathol. 30, 315-327 (2008).

17. Szanto, I. et al. Expression of NOX1, a superoxide-generating NADPH oxidase, in colon cancer and inflammatory bowel disease. J. Pathol. 207. 164-176 (2005).

18. Ha, E.M., Oh, C.T., Bae, Y.S. \& Lee, W.J. A direct role for dual oxidase in Drosophila gut immunity. Science 310, 847-850 (2005).

19. McKenzie, S.J., Baker, M.S., Buffinton, G.D. \& Doe, W.F. Evidence of oxidant-induced injury to epithelial cells during inflammatory bowel disease. J. Clin. Invest. 98, 136-141 (1996).

20. Ha, E.M. et al. Coordination of multiple dual oxidase-regulatory pathways in responses to commensal and infectious microbes in drosophila gut. Nat. Immunol. 10, 949-957 (2009).

21. Lipinski, S. et al. DUOX2-derived reactive oxygen species are effectors of NOD2-mediated antibacterial responses. J. Cell Sci. 122 (Part 19), 3522-3530 (2009).

22. Rigutto, S. et al. Activation of dual oxidases Duox1 and Duox2: differential regulation mediated by camp-dependent protein kinase and protein kinase C-dependent phosphorylation. J. Biol. Chem. 284, 6725-6734 (2009).

23. Larsson, E. et al. Analysis of gut microbial regulation of host gene expression along the length of the gut and regulation of gut microbial ecology through MyD88. Gut 61, 1124-1131 (2012).

24. Sommer, F., Adam, N., Johansson, M.E.V., Xia, L., Hansson, G.C. \& Bäckhed, F. Altered mucus glycosylation in core 1 O-glycan-deficient mice affects microbiota composition and intestinal architecture. PLoS One 9, e85254 (2014)

25. Gewirtz, A.T., Navas, T.A., Lyons, S., Godowski, P.J. \& Madara, J.L. Cutting edge: bacterial flagellin activates basolaterally expressed TLR5 to induce epithelial proinflammatory gene expression. J. Immunol. 167, 1882-1885 (2001).

26. Lee, J., Mo, J.H., Shen, C., Rucker, A.N. \& Raz, E. Toll-like receptor signaling in intestinal epithelial cells contributes to colonic homoeostasis. Curr. Opin. Gastroenterol. 23, 27-31 (2007).

27. Grasberger, H., El-Zaatari, M., Dang, D.T. \& Merchant, J.L. Dual oxidases control release of hydrogen peroxide by the gastric epithelium to prevent Helicobacter felis infection and inflammation in mice. Gastroenterology 145, 1045-1054 (2013).

28. Crosnier, C., Stamataki, D. \& Lewis, J. Organizing cell renewal in the intestine: stem cells, signals and combinatorial control. Nat. Rev. Genet. 7, 349-359 (2006).

29. Medema, J.P. \& Vermeulen, L. Microenvironmental regulation of stem cells in intestinal homeostasis and cancer. Nature 474, 318-326 (2011).

30. Kleerebezem, M. \& Vaughan, E.E. Probiotic and gut lactobacilli and bifidobacteria: molecular approaches to study diversity and activity. Annu. Rev. Microbiol. 63, 269-290 (2009).

31. Elkon, R., Linhart, C., Halperin, Y., Shiloh, Y. \& Shamir, R. Functional genomic delineation of TLR-induced transcriptional networks. BMC Genom. 8, 394 (2007). 
32. Verstrepen, L., Bekaert, T., Chau, T.L., Tavernier, J., Chariot, A. \& Beyaert, R. TLR-4, IL-1R and TNF-R signaling to NF-kappaB: variations on a common theme. Cell Mol. Life Sci. 65, 2964-2978 (2008).

33. $\mathrm{Wu}, \mathrm{Y}$. et al. Activation of TLR4 is required for the synergistic induction of dual oxidase 2 and dual oxidase A2 by IFN-gamma and lipopolysaccharide in human pancreatic cancer cell lines. J. Immunol. 190 1859-1872 (2013).

34. Park, H.S., Jung, H.Y., Park, E.Y., Kim, J., Lee, W.J. \& Bae, Y.S. Cutting edge: direct interaction of TLR4 with $\mathrm{NAD}(\mathrm{P}) \mathrm{H}$ oxidase 4 isozyme is essential for lipopolysaccharide-induced production of reactive oxygen species and activation of NF-kappa B. J. Immunol. 173, 3589-3593 (2004).

35. Kawahara, T. et al. Role of nicotinamide adenine dinucleotide phosphate oxidase 1 in oxidative burst response to Toll-like receptor 5 signaling in large intestinal epithelial cells. J. Immunol. 172, 3051-3058 (2004).

36. Asehnoune, K., Strassheim, D., Mitra, S., Kim, J.Y. \& Abraham, E. Involvement of reactive oxygen species in Toll-like receptor 4-dependent activation of NF-kappa B. J. Immunol. 172, 2522-2529 (2004).

37. Fang, F.C. Antimicrobial actions of reactive oxygen species. MBio 2, pii: e00141-11 (2011).

38. Cabiscol, E., Tamarit, J. \& Ros, J. Oxidative stress in bacteria and protein damage by reactive oxygen species. Int. Microbiol. 3, 3-8 (2000).
39. Kumar, A. etal. Commensal bacteria modulate cullin-dependent signaling via generation of reactive oxygen species. EMBO J 26, 4457-4466 (2007).

40. Kumar, A., Wu, H., Collier-Hyams, L.S., Kwon, Y.M., Hanson, J.M. \& Neish, A.S. The bacterial fermentation product butyrate influences epithelial signaling via reactive oxygen species-mediated changes in cullin-1 neddylation. J. Immunol. 182, 538-546 (2009).

41. Wichmann, A. et al. Microbial modulation of energy availability in the colon regulates intestinal transit. Cell Host Microbe 14, 582-590 (2013).

42. Johansson, M.E. et al. Bacteria penetrate the inner mucus layer before inflammation in the dextran sulfate colitis model. PLoS One 5, e12238 (2010).

43. Stappenbeck, T.S., Hooper, L.V., Manchester, J.K., Wong, M.H. \& Gordon, J.I. Laser capture microdissection of mouse intestine: characterizing $\mathrm{mRNA}$ and protein expression, and profiling intermediary metabolism in specified cell populations. Methods Enzymol 356, 167-196 (2002).

44. Cheng, G., Salerno, J.C., Cao, Z., Pagano, P.J. \& Lambeth, J.D. Identification and characterization of VPO1, a new animal heme-containing peroxidase. Free Radic. Biol. Med. 45, 1682-1694 (2008).

45. Sandelin, A., Wasserman, W.W. \& Lenhard, B. ConSite: web-based prediction of regulatory elements using cross-species comparison. Nucleic Acids Res. 32, W249-W252 (2004). 